\title{
GROWTH AND ASYMPTOTIC SETS OF SUBHARMONIC FUNCTIONS II
}

\author{
JANG-MEI WU
}

\begin{abstract}
We study the relation between the growth of a subharmonic function in the half space $\mathbb{R}_{+}^{n+1}$ and the size of its asymptotic set. In particular, we prove that for any $n \geq 1$ and $0<\alpha \leq n$, there exists a subharmonic function $u$ in the $\mathbb{R}_{+}^{n+1}$ satisfying the growth condition of order $\alpha: u(x) \leq x_{n+1}^{-\alpha}$ for $0<x_{n+1}<1$, such that the Hausdorff dimension of the asymptotic set $\bigcup_{\lambda \neq-\infty} A(\lambda)$ is exactly $n-\alpha$. Here $A(\lambda)$ is the set of boundary points at which $f$ tends to $\lambda$ along some curve. This proves the sharpness of a theorem due to Berman, Barth, Rippon, Sons, Fernández, Heinonen, Llorente and Gardiner cumulatively.
\end{abstract}

A function $f$ defined in a domain $D$ is said to have an asymptotic value $b \in[-\infty, \infty]$ at a point $a \in \partial D$ provided that there exists a path $\gamma$ in $D$ ending at $a$ so that $u(p)$ tends to $b$ as $p$ tends to $a$ along $\gamma$. The set of all points on $\partial D$ at which $f$ has an asymptotic value $b$ is denoted by $A(f, b)$ and called the asymptotic set for the value $b$.

G. R. MacLane [M1], [M2] studied the class of analytic functions in the unit disk having asymptotic values at a dense subset of the unit circle. Hornblower studied the analogous class of subharmonic functions. Since then, many have worked on problems of the following nature: for a subharmonic function $u$ of a certain growth, if $A(u,+\infty)$ is a small set, then $u$ has nice boundary behavior on a large set. Denote by $\mathbb{R}_{+}^{n+1}=$ $\left\{(x, y): x=\left(x_{1}, \ldots, x_{n}\right) \in \mathbb{R}^{n}, y>0\right\}$ the upper half space in $\mathbb{R}^{n+1}$. For $\alpha>0$, denote by $\mathcal{M}_{\alpha}$ the class of subharmonic functions $u$ in $\mathbb{R}_{+}^{n+1}$ which satisfy the growth condition:

$$
u(x, y) \leq C(u) y^{-\alpha} \quad \text { for } \quad 0<y<1
$$

for some constant $C(u)>0$. 
Denote by $\mathcal{F}(u)$ the Fatou set consisting of points on $\partial \mathbb{R}_{+}^{n+1}$ at which $u$ has finite vertical limits. For $\beta>0$, denote by $H^{\beta}$ the $\beta$-dimensional Hausdorff content.

The following theorem is due to Berman, Barth, Rippon, Sons, Fernández, Heinonen, Llorente and Gardiner cumulatively, see $[\mathbf{F H L}]$ and $[\mathbf{G}]$.

Theorem A. Let $n \geq 1,0<\alpha \leq n$ and $u$ be a subharmonic function in the class $\mathcal{M}_{\alpha}$. Let $B$ be a ball on $\partial \mathbb{R}_{+}^{n+1}$. If

$$
H^{n-\alpha}(A(u,+\infty) \cap B)<H^{n-\alpha}(B),
$$

then $H^{n}(\mathcal{F}(u) \cap B)>0$.

Denote by $A^{\prime}(u)=\bigcup_{-\infty<b \leq+\infty} A(u, b)$. Theorem A implies the following.

Theorem B. Let $n \geq 1,0<\alpha \leq n$ and $u \in \mathcal{M}_{\alpha}$. Then

$$
\operatorname{dim}\left(A^{\prime}(u)\right) \geq n-\alpha .
$$

We prove in this note that Theorem B is sharp.

Theorem 1. Given $n \geq 1$ and $0<\alpha \leq n$, there exists a subharmonic function $u$ in $\mathcal{M}_{\alpha}$ so that

$$
\operatorname{dim}\left(A^{\prime}(u)\right)=n-\alpha .
$$

It has been proved ([FHL], $[\mathbf{W}])$ that for $0<\alpha \leq 1$, there exists a harmonic function $h$ in $\mathcal{M}_{\alpha}$ such that

$$
\operatorname{dim}\left(A^{\prime}(h)\right)=n-\alpha .
$$

In general, there is more flexibility in constructing subharmonic functions than harmonic functions. In the proof of Theorem 1, we assign values of $u$ on a grid in $\mathbb{R}_{+}^{n+1}$ to force $u$ to have the desired growth, and shift the cumbersome work to the proof of subharmonicity. In order to construct such harmonic functions, we need to assure the harmonicity before regulating the growth. Our attempts have been unsuccessful when $1<\alpha \leq n$ and it is not clear whether $n-\alpha$ is the critical dimension in the harmonic case.

We proceed to prove Theorem 1 for $n \geq 2$, using ideas from [FHL] and $[\mathbf{W}]$.

From now on, assume $n \geq 2$ and $0<\alpha \leq n$; and denote by $C, C_{1}, C_{2} \ldots$ positive constants depending at most on $n$ and $\alpha$, with actual values of $C$ varying from line to line. 


\section{Two lemmas.}

Let $L$ be a cylindrical set of the form $\{(x, y): x \in S$ and $c<y<d\}$ or $\{(x, y): x \in S$ and $c \leq y \leq d\}$, with $S \subseteq \mathbb{R}^{n}$ and $c, d \in \mathbb{R}^{1}$. Denote by $L^{t}, L^{s}$ and $L^{b}$, the top $\partial L \cap\{y=d\}$, the side $\partial L \cap\{c<y<d\}$ and the bottom $\partial L \cap\{y=c\}$ of $L$ respectively.

Lemma 1. Let $r>0$ and $D, Q$ be two cubes in $\mathbb{R}^{n+1}$ defined by

$$
D=\left\{(x, y): \sup \left|x_{j}\right|<2 r, 0<y<4 r\right\}
$$

and

$$
Q=\left\{(x, y): \sup \left|x_{j}\right|<4 r, 0<y<8 r\right\},
$$

let $E=\left\{(x, 0): \sup \left|x_{j}\right|<r / 2\right\}$ and $F=\left\{(x, 0): \sup \left|x_{j}\right|<3 r / 2\right\}$. Let $G$ be the Green function for $Q$, and $\omega^{(x, y)}(S, Q)$ be the harmonic measure of a set $S$ on $\partial Q$ with respect to $Q$ evaluated at the point $(x, y) \in Q$. Then there exist constants $C_{1}, C_{2}$ and $C$ such that

$$
\omega^{(x, y)}(E, Q)>C_{1} \quad \text { for } \quad(x, y) \in D^{t},
$$

(4) $\omega^{(x, y)}(E, Q)>C_{2} \omega^{(x, y)}\left(F \cup Q^{t} \cup Q^{s}, Q\right) \quad$ for $\quad(x, y) \in D^{t} \cup D^{s}$,

$$
C^{-1}(8 r-y) r^{-n-1} \leq \frac{\partial G}{\partial n}\left((x, y),\left(x^{\prime}, 0\right)\right) \leq C(8 r-y) r^{-n-1}
$$

for $\sup \left|x_{j}\right|<2 r, 4 r<y<8 r$ and $\left(x^{\prime}, 0\right) \in D^{b}$, and $n$ the unit inward normal at $\left(x^{\prime}, 0\right)$.

Lemma 2. Let $u$ be a function continuous in $B=\left\{(x, y): \Sigma x_{j}^{2}+\right.$ $\left.y^{2}<r^{2}\right\}$ and harmonic in $B \backslash\{y=0\}$ with first partials continuous on $B \cap\{y \geq 0\}$ and also on $B \cap\{y \leq 0\}$. If the left and right partials satisfy

$$
\left(\frac{\partial u}{\partial y}\right)_{-}(x, 0) \leq\left(\frac{\partial u}{\partial y}\right)_{+}(x, 0) \quad \text { on } \quad B \cap\{y=0\},
$$

then $u$ is subharmonic in $B$.

Both lemmas are elementary. The precise statement of Lemma 2 can be found in $[\mathbf{D}]$.

\section{A partition of $\mathbb{R}_{+}^{n+1}$.}

Choose and fix an odd integer $R$ :

$$
R>\max \left\{10^{5 n}, 10^{5 n / \alpha}, 2 C_{1}^{-1}, C_{2}^{-1},\left(2 C_{3} / C_{4}\right)^{2 / \alpha}\right\},
$$


where $C_{1}$ and $C_{2}$ are constants from Lemma $1, C_{3}$ and $C_{4}$ are to be specified later. Choose for $k \geq 1, \delta_{k}$ so that

$$
2 \delta_{k} R^{2 k} \quad \text { is an odd integer }
$$

and

$$
\left(k+R^{3 \alpha / 2 n}\right) R^{-2 k \alpha / n} \leq \delta_{k} \leq 2\left(k+R^{3 \alpha / 2 n}\right) R^{-2 k \alpha / n} .
$$

Note from (6) that $R^{-\alpha / n}<10^{-5}$, thus $\delta_{k}<\frac{1}{100}$. Let for $k \geq 1$,

$$
r_{k}=\delta_{k} R^{-k^{2}}
$$

Denote by $A$ the collection of all integer lattice points on $\mathbb{R}^{n}$ and by

$$
A_{k}=\left\{R^{-k^{2}} a: a \in A\right\}
$$

For $k \geq 1$ and $a \in A_{k}$, let

$$
\begin{aligned}
\Gamma_{k, a} & =\left\{(x, y): r_{k+1} \leq y \leq r_{k} \text { and } \sup _{1 \leq j \leq n}\left|x_{j}-a_{j}\right| \leq r_{k}\right\} \\
\Gamma_{k} & =\cup_{a} \Gamma_{k, a} \\
\Omega_{k} & =\left\{(x, y): r_{k+1}<y<r_{k}\right\} \backslash \Gamma_{k},
\end{aligned}
$$

and let

$$
\Omega_{0}=\left\{(x, y): y>r_{1}\right\}
$$

Note that each $\Omega_{k}$ is connected because $n \geq 2$, that sets in $\left\{\Gamma_{k}\right\}_{k \geq 1} \cup$ $\left\{\Omega_{k}\right\}_{k \geq 0}$ have mutually disjoint interiors and that

$$
\bigcup_{k \geq 1} \Gamma_{k} \cup \bigcup_{k \geq 0} \bar{\Omega}_{k}=\mathbb{R}_{+}^{n+1} .
$$

The top $\Gamma_{k, a}^{t}$ of each $\Gamma_{k, a}$ is either completely contained in $\Gamma_{k-1}^{b}$ or completely contained in $\Omega_{k-1}^{b}$. To prove this, we claim that $\left(\Gamma_{k-1}^{b} \cap \Omega_{k-1}^{b}\right) \cap \Gamma_{k}^{t}=\emptyset$. Suppose that $\left(x, r_{k}\right) \in \Gamma_{k-1}^{b} \cap \Omega_{k-1}^{b}$, then $\left|x_{j}-p R^{-(k-1)^{2}}\right|=\delta_{k-1} R^{-(k-1)^{2}}$ for some integers $j(1 \leq j \leq n)$ and $p$. To see that $\left(x, r_{k}\right) \notin \Gamma_{k}^{t}$, it is enough to show that $\left|x_{j}-q R^{-k^{2}}\right|>\delta_{k} R^{-k^{2}}$ for all integers $q$; or equivalently, $\left|p R^{2 k-1} \pm \delta_{k-1} R^{2 k-1}-q\right|>\delta_{k}$ for all integers $q$. Because $\delta_{k}<\frac{1}{100}$ and $2 \delta_{k-1} R^{2 k-1}$ is an odd integer, the inequality follows. 
For each $k \geq 0$, denote by $H_{k}$ the set consisting of $\bar{\Omega}_{k}$ and those $\Gamma_{j, a}$ $\left(j>k\right.$ and $\left.a \in A_{j}\right)$ that can be connected to $\Omega_{k}$ by paths not intersecting $\Omega_{k^{\prime}}$ for any $k^{\prime} \neq k$. That is, $\Gamma_{j, a} \subseteq H_{k}$ if and only if $j>k$ and the line segment $\left\{x=a, \frac{r_{j}}{2} \leq y \leq \frac{r_{k}}{2}\right\}$ is contained in $\Omega_{k} \cup\left(\cup\left\{\Gamma_{i}: i>k\right\}\right)$. Note from the comment in the last paragraph, the interiors of $H_{k}$ 's are mutually disjoint and that

$$
\bigcup_{k \geq 0} H_{k}=\mathbb{R}_{+}^{n+1}
$$

\section{Size of the asymptotic sets.}

Suppose that $u$ is a function in $\mathcal{M}_{\alpha}$ that satisfies

$$
\lim _{k \rightarrow \infty} \sup \left\{u(x, y):(x, y) \in\left(\bigcup_{j \geq 0} \partial H_{j}\right) \bigcap\left\{y \leq r_{k}\right\}\right\}=-\infty .
$$

Then any asymptotic path $\gamma$, along which $u$ has an asymptotic value $b \neq-\infty$, does not meet $\cup_{j \geq 0} \partial H_{j} \bigcap\{0<y<t\}$ for some $t>0$; therefore $\gamma \cap\{0<y<t / 2\}$ is contained in a certain $H_{k}$. From this, it follows that

$$
A^{\prime}(u) \subseteq \bigcup_{k \geq 1}\left(\bigcap_{j \geq k} \Gamma_{j}^{*}\right)
$$

where $\Gamma_{j}^{*}$ is the projection of $\Gamma_{j}$ onto $\partial \mathbb{R}_{+}^{n+1}$. Let $T$ be a unit cube on $\mathbb{R}^{n}$ and $k$ a positive integer. Given $K>k$, the set $T \cap\left(\cap_{j \geq k} \Gamma_{j}^{*}\right)$ can be covered by at most

$$
\begin{aligned}
N & \equiv C(k)\left(\frac{1}{R^{-k^{2}}} \cdot \frac{2 r_{k}}{R^{-(k+1)^{2}}} \cdots \frac{2 r_{K-1}}{R^{-K^{2}}}\right)^{n} \\
& \equiv C(k)\left(2 \delta_{k}\right)^{n}\left(2 \delta_{k+1}\right)^{n} \ldots\left(2 \delta_{K-1}\right)^{n} R^{K^{2} n}
\end{aligned}
$$

cubes in $T$ of side length $2 \delta_{K} R^{-K^{2}}$ each. If $n \geq \beta>n-\alpha$, then in view of (8),

$$
\begin{aligned}
& N \cdot\left(2 \delta_{K} R^{-K^{2}}\right)^{\beta} \\
& \quad \leq C(n, R, \alpha, \beta, k) 4^{n K}\left(\left(K+R^{2}\right) !\right)^{n} R^{K^{2}(n-\alpha-\beta)} R^{2 K(n-\beta) \alpha / n}
\end{aligned}
$$

which approaches 0 as $K \rightarrow \infty$. This implies that $\operatorname{dim}\left(A^{\prime}(u)\right) \leq n-\alpha$. In view of $(1), \operatorname{dim}\left(A^{\prime}(u)\right)=n-\alpha$. 
Construction of the function $u$.

Now we are ready to construct a subharmonic $u$ in $\mathcal{M}_{\alpha}$ that has the property (9). Let for $k \geq 0$,

$$
M_{k}=R^{\alpha k^{2}} \quad \text { and } \quad m_{k}=R^{-1+\alpha k^{2}}
$$

and let for $\lambda>0$,

$$
\lambda \Gamma_{k, a}^{t}=\left\{\left(x, r_{k}\right): \sup _{j}\left|x_{j}-a_{j}\right| \leq \lambda r_{k}\right\}
$$

and

$$
\lambda \Gamma_{k}^{t}=\bigcup_{a} \lambda \Gamma_{k, a}^{t}
$$

Define $u$ on $\left(\cup_{k \geq 1} \partial \Gamma_{k}\right) \cup\left(\cup_{k \geq 0} \partial \Omega_{k}\right) \equiv\left(\cup_{k \geq 1} \Gamma_{k}^{s}\right) \cup\left(\cup_{k \geq 1}\left\{y=r_{k}\right\}\right)$ as follows: for each $k \geq 1$

$$
u=-m_{k} \quad \text { on } \quad \Gamma_{k}^{s},
$$

and $u$ is $C^{2}$ on $\left\{y=r_{k}\right\}$ with values

$$
\begin{aligned}
u=M_{k} \quad \text { on } \quad \frac{1}{2} \Gamma_{k}^{t}, \\
-m_{k} \leq u \leq M_{k} \quad \text { on } \quad \frac{3}{4} \Gamma_{k}^{t} \backslash \frac{1}{2} \Gamma_{k}^{t}, \\
u=-m_{k} \quad \text { on } \quad \frac{5}{4} \Gamma_{k}^{t} \backslash \frac{3}{4} \Gamma_{k}^{t}, \\
-m_{k} \leq u \leq-m_{k-1} \quad \text { on } \quad \frac{3}{2} \Gamma_{k}^{t} \backslash \frac{5}{4} \Gamma_{k}^{t}, \\
u=-m_{k-1} \quad \text { on } \quad\left\{y=r_{k}\right\} \backslash \frac{3}{2} \Gamma_{k}^{t},
\end{aligned}
$$

with partial derivatives

$$
\Sigma_{j}\left|\frac{\partial}{\partial x_{j}} u\right| \leq C M_{k} r_{k}^{-1},
$$

and

$$
\Sigma_{i, j}\left|\frac{\partial^{2}}{\partial x_{i} \partial x_{j}} u\right| \leq C M_{k} r_{k}^{-2},
$$

for some constant $C$. 
Extend $u$ to be continuous on $\mathbb{R}_{+}^{n+1}$, bounded on $\Omega_{0}$, harmonic in each $\Omega_{k}(k \geq 0)$ and harmonic in the interior of each $\Gamma_{k}(k \geq 1)$. By the maximum principle,

$$
\begin{aligned}
-m_{1} \leq u \leq M_{1} & \text { on } \quad\left\{y \geq r_{1}\right\} \\
-m_{k+1} \leq u \leq M_{k+1} & \text { on } \quad\left\{r_{k+1} \leq y \leq r_{k}\right\} \quad(k \geq 1)
\end{aligned}
$$

Note from the definition of $u$ that $u$ is negative on $\cup \partial H_{j}$ and

$$
u \leq-m_{k-1} \quad \text { on } \quad\left(\bigcup_{j \geq 0} \partial H_{j}\right) \bigcap\left\{y \leq r_{k}\right\} \quad(k \geq 1) \text {. }
$$

Since $\left\{m_{k}\right\}$ is unbounded, $u$ satisfies (9). Therefore (2) holds for $u$.

\section{Subharmonicity.}

The subharmonicity is proved by induction. Note that $u$ is harmonic in $\left\{y>r_{1}\right\}$, and suppose that $u$ is subharmonic in $\left\{y>r_{k}\right\}$ for some $k \geq 1$. In order to prove that $u$ is subharmonic in $\left\{y>r_{k+1}\right\}$, we need to verify the submean value property on $\Gamma_{k}^{s} \cup\left\{y=r_{k}\right\}$. We shall prove that $u$ has a local minimum at each point in $\Gamma_{k}^{s} \cup\left(\frac{5}{4} \Gamma_{k}^{t} \backslash \frac{3}{4} \Gamma_{k}^{t}\right)$, and compare the normal derivatives from both sides on the remaining part and then use Lemma 2.

First we give estimates of $u$ on some subsets of $\Omega_{k}$ and $\Gamma_{k}$.

For $k \geq 1$ and $a \in A_{k}$, let

$$
\begin{aligned}
D_{k, a} & =\left\{(x, y): \sup _{j}\left|x_{j}-a_{j}\right|<2 r_{k}, r_{k}<y<5 r_{k}\right\}, \\
Q_{k, a} & =\left\{(x, y): \sup _{j}\left|x_{j}-a_{j}\right|<4 r_{k}, r_{k}<y<9 r_{k}\right\}, \\
D_{k} & =\bigcup_{a} D_{k, a} \text { and } Q_{k}=\bigcup_{a} Q_{k, a} .
\end{aligned}
$$

Note from (6) and (8) that $\left\{Q_{k, a}: a \in A_{k}\right\}$ are mutually disjoint and that

$$
Q_{k} \subseteq\left\{r_{k}<y<r_{k-1}\right\} \backslash \partial \Gamma_{k-1} .
$$

Let for $k \geq 0$,

$$
\Omega_{k}^{\prime}=\Omega_{k} \backslash \bar{D}_{k+1},
$$

and for $k \geq 1$,

$$
\Gamma_{k}^{\prime}=\Gamma_{k} \backslash \bar{D}_{k+1}
$$


Lemma 3. For $k \geq 1$,

$$
u>C M_{k} \text { on } D_{k}^{t},
$$

and

$$
u>-m_{k} \quad \text { on } \quad \Omega_{k}^{\prime} \cup \Gamma_{k}^{\prime} .
$$

Proof: Let $k \geq 2$ and recall that $-m_{k} \leq u \leq M_{k}$ in $\left\{r_{k} \leq y \leq r_{k-1}\right\}$ and $Q_{k, a} \subseteq\left\{r_{k} \leq y \leq r_{k-1}\right\}$. Apply the maximum principle to $u$ on $Q_{k, a}$, we have

$$
u(x, y) \geq M_{k} \omega^{(x, y)}\left(\frac{1}{2} \Gamma_{k, a}^{t}, Q_{k, a}\right)-m_{k}\left(1-\omega^{(x, y)}\left(\frac{1}{2} \Gamma_{k, a}^{t}, Q_{k, a}\right)\right)
$$

for $(x, y) \in Q_{k, a}$, where $\omega$ is the harmonic measure. In view of (3), (6) and (10)

$$
u(x, y) \geq M_{k} C_{1}-m_{k}\left(1-C_{1}\right)>M_{k} C_{1} / 2
$$

for $(x, y) \in D_{k, a}^{t}$. This proves (14).

Next consider $u$ on $\Omega_{k-1}$, and note that $u \geq-m_{k-1}$ on $\partial \Omega_{k-1} \backslash \frac{3}{2} \Gamma_{k}^{t}$, $-m_{k} \leq u \leq M_{k}$ on $\frac{3}{2} \Gamma_{k}^{t}$ and $u=M_{k}$ on $\frac{1}{2} \Gamma_{k}^{t}$. Apply the maximum principle to $m_{k-1}+u$ on $\Omega_{k-1}$, we obtain

$$
\begin{aligned}
m_{k-1}+u(x, y) \geq \Sigma^{\prime}\left(M_{k} \omega^{(x, y)}\right. & \left(\frac{1}{2} \Gamma_{k, a}^{t}, \Omega_{k-1}\right) \\
& \left.-m_{k} \omega^{(x, y)}\left(\frac{3}{2} \Gamma_{k, a}^{t} \backslash \frac{1}{2} \Gamma_{k, a}^{t}, \Omega_{k-1}\right)\right)
\end{aligned}
$$

for $(x, y) \in \Omega_{k-1}$, where $\Sigma^{\prime}$ sums over those $a \in A_{k}$ such that $Q_{k, a} \subseteq$ $\Omega_{k-1}$ and $\omega$ is the harmonic measure. If $Q_{k, a} \subseteq \Omega_{k-1}$ and $(x, y) \in Q_{k, a}$, then

$$
\omega^{(x, y)}\left(\frac{1}{2} \Gamma_{k, a}^{t}, \Omega_{k-1}\right) \geq \omega^{(x, y)}\left(\frac{1}{2} \Gamma_{k, a}^{t}, Q_{k, a}\right)
$$

and

$$
\omega^{(x, y)}\left(\frac{3}{2} \Gamma_{k, a}^{t} \backslash \frac{1}{2} \Gamma_{k, a}^{t}, \Omega_{k-1}\right) \leq \omega^{(x, y)}\left(\frac{3}{2} \Gamma_{k, a}^{t} \cup Q_{k, a}^{t} \cup Q_{k, a}^{s}, Q_{k, a}\right)
$$

by the maximum principle. It follows from (4), (6) and (10) that

$$
\omega^{(x, y)}\left(\frac{3}{2} \Gamma_{k, a}^{t} \cup Q_{k, a}^{t} \cup Q_{k, a}^{s}, Q_{k, a}\right) \leq \frac{M_{k}}{m_{k}} \omega^{(x, y)}\left(\frac{1}{2} \Gamma_{k, a}^{t}, Q_{k, a}\right)
$$


for $(x, y)$ in $D_{k, a}^{t} \cup D_{k, a}^{s}$. Hence $u \geq-m_{k-1}$ on $D_{k, a}^{t} \cup D_{k, a}^{s}$. Since $u \geq$ $-m_{k-1}$ on $\partial \Omega_{k-1}^{\prime} \backslash \bar{D}_{k}, u>-m_{k-1}$ in $\Omega_{k-1}^{\prime}$ by the maximum principle. Therefore $u>-m_{k}$ in $\Omega_{k}^{\prime}$ for $k \geq 1$. The estimate on $\Gamma_{k}^{\prime}$ follows by a similar argument.

This completes the proof of Lemma 3.

From (13), (15) and the monotonicity of $m_{k}$, it follows that $u \geq-m_{k}$ on $\overline{\Omega_{k}^{\prime}} \cup \overline{\Gamma_{k}^{\prime}} \cup\left\{y \geq r_{k}\right\}$. Therefore at each point in $\Gamma_{k}^{s} \cup\left(\frac{5}{4} \Gamma_{k}^{t} \backslash \frac{3}{4} \Gamma_{k}^{t}\right), u$ has a local minimum $-m_{k}$, thus the submean value property.

In view of Lemma 2 , to prove the subharmonicity on $\left\{y=r_{k}\right\} \backslash$ $\left(\frac{5}{4} \Gamma_{k}^{t} \backslash \frac{3}{4} \Gamma_{k}^{t}\right)$, it suffices to prove

$$
\left(\frac{\partial u}{\partial y}\right)_{-} \leq\left(\frac{\partial u}{\partial y}\right)_{+} \quad \text { on } \quad\left\{y=r_{k}\right\} \backslash\left(\frac{5}{4} \Gamma_{k}^{t} \backslash \frac{3}{4} \Gamma_{k}^{t}\right)
$$

Because of (15), $u \geq-m_{k-1}$ in $\left\{r_{k} \leq y \leq r_{k-1}\right\} \backslash \overline{D_{k}}$. Since $u=$ $-m_{k-1}$ on $\left\{y=r_{k}\right\} \backslash 2 \Gamma_{k}^{t}$,

$$
\left(\frac{\partial u}{\partial y}\right)_{+} \geq 0 \quad \text { on } \quad\left\{y=r_{k}\right\} \backslash 2 \Gamma_{k}^{t} .
$$

We claim that

$$
\left|\left(\frac{\partial u}{\partial y}\right)_{+}\right| \leq C_{3} M_{k} r_{k}^{-1} \text { on } 2 \Gamma_{k}^{t}
$$

for some constant $C_{3}>0$ depending only on $n$. To this end, fix $a \in$ $A_{k}$ and let $g$ be a $C^{2}$ function in a neighborhood of $\overline{Q_{k, a}}$, with values $g\left(x, r_{k}\right)=u\left(x, r_{k}\right)$ and $g(x, y)=-m_{k-1}$ in $Q_{k, a} \backslash D_{k, a}$,

$$
|\operatorname{grad} g| \leq C M_{k} r_{k}^{-1}
$$

and

$$
|\triangle g| \leq C M_{k} r_{k}^{-2}
$$

on $\overline{Q_{k, a}}$. This is possible because $u$ satisfies (11) and (12). Let $h$ be a function continuous on $\overline{Q_{k, a}}$, harmonic in $Q_{k, a}$ with boundary values $h(x, y)=0$ on $\partial Q_{k, a} \cap\left\{y=r_{k}\right\}$ and $h(x, y)=u(x, y)+m_{k-1}$ on $\partial Q_{k, a} \cap$ $\left\{y>r_{k}\right\}$. Since $\triangle(u-h)=0$ in $Q_{k, a}$ and $u-h=g$ on $\partial Q_{k, a}$, a boundary estimate of derivatives $\left[\mathbf{P W}\right.$, p. 144] shows that in $Q_{k, a}$,

$$
|\operatorname{grad}(u-h)| \leq \max _{\partial Q_{k, a}}|\operatorname{grad} g|+\max _{\bar{Q}_{k, a}}|\triangle g| \cdot \operatorname{diam} Q_{k, a} .
$$


Therefore $\left|\left(\frac{\partial u}{\partial y}\right)_{+}\right| \leq\left|\left(\frac{\partial h}{\partial y}\right)_{+}\right|+C M_{k} r_{k}^{-1}$ on $D_{k, a}^{b}$. Since $0<h<M_{k}$ in $Q_{k, a}$ and $h=0$ on $Q_{k, a}^{b},\left|\left(\frac{\partial h}{\partial y}\right)_{+}\right| \leq C M_{k} r_{k}^{-1}$ on $\bar{D}_{k, a}^{b}$. From these, estimate (18) follows.

To obtain an upper bound for $\left(\frac{\partial u}{\partial y}\right)_{-}$on $\left\{y=r_{k}\right\} \backslash\left(\frac{5}{4} \Gamma_{k}^{t} \backslash \frac{3}{4} \Gamma_{k}^{t}\right)$, we write $u=w+v$ in $\left\{5 r_{k+1} \leq y \leq r_{k}\right\}$, where $w$ is bounded and harmonic in $\left\{5 r_{k+1}<y<r_{k}\right\} \backslash \Gamma_{k}^{s}$ with boundary values $w=u$ on $\left(\Gamma_{k}^{s} \cap\{y>\right.$ $\left.\left.5 r_{k+1}\right\}\right) \cup\left\{y=r_{k}\right\}$ and $w=-m_{k}$ on $\left\{y=5 r_{k+1}\right\}$. Note from (15) and the maximum principle that $-m_{k} \leq w \leq M_{k}$. Following the argument for (18), we obtain

$$
\left|\left(\frac{\partial w}{\partial y}\right)_{-}\right| \leq C_{3} M_{k} r_{k}^{-1} \quad \text { on } \quad\left\{y=r_{k}\right\} \backslash\left(\frac{5}{4} \Gamma_{k}^{t} \backslash \frac{3}{4} \Gamma_{k}^{t}\right) ;
$$

if necessary, we shall replace $C_{3}$ in (18) by a larger number. Recall from (15) that $u \geq-m_{k}$ in $\left\{5 r_{k+1}<y<r_{k}\right\}$, thus $v$ is nonnegative; and note from (14) that

$$
v>u>C M_{k+1} \quad \text { on } \quad D_{k+1}^{t} .
$$

Given a point $\left(x^{\prime}, r_{k}\right) \in\left\{y=r_{k}\right\} \backslash\left(\frac{5}{4} \Gamma_{k}^{t} \backslash \frac{3}{4} \Gamma_{k}^{t}\right)$, let

$$
U=\left\{(x, y): \sup \left|x_{j}-x_{j}^{\prime}\right|<r_{k} / 8 \quad \text { and } \quad 5 r_{k+1} \leq y \leq r_{k}\right\}
$$

and note that $U$ does not meet $\Gamma_{k}^{s}$. Denote by $G$ the Green function for $U$. If $\frac{2}{3} r_{k}<y<r_{k}$, then

$$
\begin{aligned}
v\left(x^{\prime}, y\right) & \geq C M_{k+1} \omega^{\left(x^{\prime}, y\right)}\left(D_{k+1}^{t} \cap \partial U, U\right) \\
& \geq C M_{k+1} \int_{D_{k+1}^{t} \cap \partial U} \frac{\partial G}{\partial n}\left(\left(x^{\prime}, y\right),\left(x, 5 r_{k+1}\right)\right) d x .
\end{aligned}
$$

Because $D_{k+1}^{t} \cap \partial U \cap\left\{y=5 r_{k+1}\right\}$ has $n$-dimensional measure $\geq C \delta_{k+1}^{n} r_{k}^{n}$, from (5) and the maximum principle it follows that

$$
v\left(x^{\prime}, y\right) \geq C M_{k+1}\left(r_{k}-y\right) r_{k}^{-n-1} \delta_{k+1}^{n} r_{k}^{n} .
$$

Because $v\left(x^{\prime}, r_{k}\right)=0$,

$$
\left(\frac{\partial v}{\partial y}\right)_{-}\left(x^{\prime}, r_{k}\right) \leq-C_{4} M_{k+1} \delta_{k+1}^{n} r_{k}^{-1}
$$

for some constant $C_{4}$. 
Combining estimates (17) through (20), we conclude that on $\{y=$ $\left.r_{k}\right\} \backslash\left(\frac{5}{4} \Gamma_{k}^{t} \backslash \frac{3}{4} \Gamma_{k}^{t}\right)$,

$$
\left(\frac{\partial u}{\partial y}\right)_{-}-\left(\frac{\partial u}{\partial y}\right)_{+} \leq-C_{4} M_{k+1} \delta_{k+1}^{n} r_{k}^{-1}+2 C_{3} M_{k} r_{k}^{-1},
$$

which is negative in view of (6), (8) and (10). This proves (16).

Hence $u$ is subharmonic in $\mathbb{R}_{+}^{n+1}$.

\section{Growth estimates.}

Let $k \geq 1$. Because $-m_{k+1} \leq u \leq M_{k+1}$ in $\left\{r_{k+1} \leq y \leq r_{k}\right\}$ and $5 r_{k+1}<R^{-(k+1)^{2}}<r_{k}$, we have

$$
u(x, y) \leq M_{k+1}=R^{\alpha(k+1)^{2}} \leq y^{-\alpha} \quad \text { for } \quad r_{k+1} \leq y \leq R^{-(k+1)^{2}} .
$$

Let $\omega$ be the harmonic measure of $\frac{3}{4} \Gamma_{k+1}^{t}$ with respect to the half space $\left\{y>r_{k+1}\right\}$. Then

$$
\omega(x, y) \leq C \delta_{k+1}^{n} \quad \text { for } \quad y>R^{-(k+1)^{2}} .
$$

By (6), (8), (10) and the maximum principle,

$$
\begin{aligned}
u(x, y) & \leq M_{k}+M_{k+1} \omega(x, y) \leq M_{k}+C M_{k+1} \delta_{k+1}^{n} \\
& <C(n, \alpha, R) r_{k}^{-\alpha}<C(n, \alpha, R) y^{-\alpha}
\end{aligned}
$$

for $R^{-(k+1)^{2}}<y<r_{k}$. Because $u$ is bounded in $\left\{y>r_{1}\right\}$,

$$
u(x, y) \leq C(u) y^{-\alpha} \quad \text { for } \quad 0<y<1 .
$$

This completes the proof of Theorem 1.

\section{References}

[D] D. Drasin, Some problems related to MacLane class, J. London Math. Soc. 23 (1981), 280-286.

[FHL] J. L. Fernández, J. Heinonen and J. G. Llorente, Asymptotic values of subharmonic functions, Proc. London Math. Soc. 73 (1996), 404-430.

[G] S. J. Gardiner, Asymptotic values and minimal fine limits of subharmonic functions of slow growth, Preprint. 
[M1] G. R. MacLane, Holomorphic functions, of arbitrarily slow growth, without radial limits, Michigan Math. J. 9 (1962), 21-24.

[M2] G. R. MacLane, Asymptotic values of holomorphic functions, Rice Univ. Stud. 49. (1963) 83 pp.

[PW] M. H. Protter and H. F. Weinberger, Maximum Principles in Differential Equations, Prentice-Hall Inc., 1967.

[W] J.-M. WU, Growth and Asymptotic sets of subharmonic functions, J. London Math. Soc. (to appear).

\author{
Department of Mathematics \\ University of Illinois \\ 1409 West Green Street \\ Urbana, Illinois 61801 \\ U.S.A. \\ e-mail:wu@math.uiuc.edu
}

Primera versió rebuda el 22 d'octubre de 1997, darrera versió rebuda el 7 de maig de 1998 